\title{
Nonlinear magnetogyrotropic photogalvanic effect
}

\author{
H. Diehl, ${ }^{1}$ V. A. Shalygin, ${ }^{2}$ L. E. Golub, ${ }^{3}$ S. A. Tarasenko, ${ }^{3}$ S. N. Danilov, ${ }^{1}$ V. V. Bel'kov, ${ }^{1,3}$ E. G. Novik, ${ }^{4}$ H. Buhmann, ${ }^{4}$ \\ C. Brüne, ${ }^{4}$ L. W. Molenkamp,${ }^{4}$ E. L. Ivchenko, ${ }^{3}$ and S. D. Ganichev ${ }^{1}$ \\ ${ }^{1}$ Terahertz Center, University of Regensburg, 93040 Regensburg, Germany \\ ${ }^{2}$ St. Petersburg State Polytechnic University, 195251 St. Petersburg, Russia \\ ${ }^{3}$ A.F. Ioffe Physical-Technical Institute of the Russian Academy of Sciences, 194021 St. Petersburg, Russia \\ ${ }^{4}$ Physical Institute (EP3), University of Würzburg, 97074 Würzburg, Germany
}

(Received 20 May 2009; revised manuscript received 3 July 2009; published 19 August 2009)

\begin{abstract}
We report on the observation of magnetic-field-induced photocurrent in $\mathrm{HgTe} / \mathrm{HgCdTe}$ quantum wells of different widths. Both the intrasubband and interband absorption of infrared/terahertz radiation in the heterostructures is shown to cause a dc electric current in the presence of an in-plane magnetic field. The photocurrent behavior upon variation in the radiation polarization, magnetic-field strength, and temperature is studied. At a moderate magnetic field the current exhibits a linear field dependence. At high magnetic fields, however, it becomes nonlinear and is dominated by a cubic in magnetic-field contribution. The latter effect is observed in quantum wells with the inverted band structure only. The experimental results are analyzed in terms of the phenomenological theory and microscopic models of magnetogyrotropic photogalvanic effect based on asymmetry of optical transitions and/or asymmetric relaxation of carriers in the momentum space. The effect is shown to be related to the gyrotropic properties of the structures. The developed theory of magnetogyrotropic photocurrent describes well all experimental results. It is shown that both intrasubband and interband optical transitions may lead to spin-related as well as to spin-independent magnetic-field-induced photocurrents.
\end{abstract}

DOI: 10.1103/PhysRevB.80.075311

PACS number(s): 73.21.Fg, 72.25.Fe, 78.67.De, 73.63.Hs

\section{INTRODUCTION}

Much current attention in condensed-matter physics is directed toward understanding the spin-dependent phenomena both from the fundamental point of view and due to increasing interest in spintronics devices that are based not only on the electron charge but also on its spin. Conventional lowdimensional semiconductor structures fabricated of III-V, such as InAs or GaAs, and II-VI, such as CdTe, wide gap materials are in focus of present day investigations. Quantum well (QW) structures based on $\mathrm{HgTe}$ appear to be very attractive for the study of fundamental spin-orbit effects. Narrow gap HgTe-based QWs are characterized by an extraordinary large Rashba-type spin-orbit splitting, a parameter crucial for the field of spintronics because it allows an electric field control of spins, determines the spin relaxation rate, and can be utilized for all-electric spin injection. ${ }^{1}$ The lifting of spin degeneracy is caused by spin-orbit interaction due to structure and bulk inversion asymmetries which lead to Rashba and Dresselhaus spin-orbit terms in the Hamiltonian, respectively (see, e.g., Refs. 2-7). The Rashba spin splitting in HgTe-based QWs can reach values of up to $30 \mathrm{meV}$, which is several times larger than for any other semiconductor materials and can be tuned over a wide range. ${ }^{8,9}$ Last but not least, HgTe-based QWs are characterized by a highly specific band structure which, depending on the well width and temperature, can be either normal or inverted, small effective masses about $0.02-0.04 m_{0}$ (Refs. 10 and 11) and a large Landé $g$ factor of about -20 (Ref. 12). Despite the enhanced spin features, however, there has been only a low interest in the HgTe-based QWs. This can be attributed to difficulties in the fabrication of HgTe-based devices and its moderate mobilities. Recently, a significant progress has been achieved in the growth of HgTe-based QWs. These ad- vances make high mobility samples available. ${ }^{13}$ Additionally, lithographical techniques were developed which meet the special requirements of $\mathrm{HgTe}$ QWs. ${ }^{14}$

The appearance of high quality $\mathrm{HgTe} / \mathrm{HgCdTe} \mathrm{QWs}$ resulted in the observation of numerous transport, optical, and magneto-optical spin-related effects, such as large Zeeman spin splitting, ${ }^{15}$ circular photogalvanic effect, ${ }^{16,17}$ enhancement of the subband spin splitting by introducing magnetic ions in the QW structure, ${ }^{18,19}$ and the quantum spin Hall effect. ${ }^{20,21}$ The latter effect is characterized by nondissipative transport of spin-polarized electrons and has a high potential for spintronics applications.

Here, we report on the observation of the magnetogyrotropic photogalvanic effect (MPGE) in (001)-grown $\mathrm{HgTe} /$ $\mathrm{HgCdTe}$ QWs. In general, the photogalvanic effects form a class of phenomena in which the electric field of electromagnetic radiation acts upon mobile carriers and generates a dc electric current, under short-circuit condition, or a voltage, in the case of open-circuit samples. These photocurrents appear neither due to inhomogeneity of the optical excitation (or inhomogeneity of the sample in the direction of the photocurrent) nor due to the photon drag. They are allowed in noncentrosymmetric systems only and caused by the asymmetry of elementary processes of photoexcitation and/or carrier relaxation resulting in a shift of carrier distribution in the momentum space. An additional root of photogalvanic effects is provided by application of an external magnetic field which breaks the time inversion symmetry and can lead to additional mechanisms of photocurrents. The MPGE is a magnetic-field-induced photocurrent related to the gyrotropic symmetry of the system. Microscopically, this means that the effect is contributed by a mechanism based on the magnetic or spin-dependent coupling interaction described by a product of polar and axial vector components. We present experimental and theoretical studies of MPGE induced by terahertz 
as well as mid-infrared radiation. The effect was detected in a wide temperature range from liquid helium to room temperature (Ref. 22). The MPGE has so far been detected in GaAs, InAs, GaN, and Si QWs for various spectral ranges (for a review see Ref. 30). It has been shown that different microscopic mechanisms of both paramagnetic ${ }^{23,24}$ (spin dependent) and diamagnetic ${ }^{25-28}$ origins can contribute to the photocurrent. Recently, we demonstrated that MPGE provides a tool to probe the symmetry of QWs and gives the necessary feedback to reliable growth of structures with the controllable strength and sign of the structure inversion asymmetry. ${ }^{29,30}$ Thus, the observation of MPGE gives access to the material under investigation. According to the previous studies carried out on III-V-based heterostructures the MPGE current depends linearly on the magnetic-field strength $B$. To our surprise, in $\mathrm{HgTe} / \mathrm{HgCdTe}$ QWs with inverted band structure we have detected both linear and nonlinear-in- $B$ contributions. By contrast, in QWs with the normal band ordering the nonlinear-in- $B$ photocurrent is negligibly small. The paper is organized as follows. In Sec. II we give a short overview of the experimental technique. In Sec. III the experimental results are summarized. In Sec. IV we present the phenomenological theory of the MPGE and compare its results with experimental data on polarization dependences. In Secs. V and VI we show the results of the band-structure calculations and discuss experimental data in view of the microscopic background.

\section{SAMPLES AND EXPERIMENTAL TECHNIQUE}

The experiments are carried out on $\mathrm{Hg}_{0.3} \mathrm{Cd}_{0.7} \mathrm{Te} / \mathrm{HgTe} / \mathrm{Hg}_{0.3} \mathrm{Cd}_{0.7} \mathrm{Te} \mathrm{QWs}$ having four different nominal well widths, $L_{W}: 5,8,12$, and $22 \mathrm{~nm}$. Structures are molecular beam epitaxy grown on a $\mathrm{Cd}_{0.96} \mathrm{Zn}_{0.04}$ Te substrate with the surface orientation (001). Samples with the sheet density of electrons $n_{s}$ from $1 \times 10^{11} \mathrm{~cm}^{-2}$ to 2 $\times 10^{12} \mathrm{~cm}^{-2}$ and mobility in the range between $5 \times 10^{4}$ and $2 \times 10^{5} \mathrm{~cm}^{2} / \mathrm{V}$ s at $T=4.2 \mathrm{~K}$ have been studied. In order to investigate photocurrents, we have fabricated clover-shaped mesa structures of $4 \mathrm{~mm}$ diameter (see inset of Fig. 1) using electron beam lithography and dry-etching techniques. Ohmic contacts are fabricated by thermal In bonding. The contacts in clover structures are oriented along the $x \|[100]$ and $y \|[010]$ crystallographic directions. The photocurrent is measured in unbiased structures via the voltage drop across a $50 \Omega$ load resistor. Samples were mounted in an optical cryostat which allowed us to study MPGE in the temperature range from $4.2 \mathrm{~K}$ up to room temperature. An external inplane magnetic field $B$ up to \pm 7 T could be applied in the $x$ direction using a superconducting magnet.

The measurements of magnetic-field-induced photocurrents are carried out under excitation of the samples with mid-infrared and terahertz radiation at normal incidence. The geometry of the experiment is sketched in the inset in Fig. 1(a). In (001)-oriented unbiased quantum well structures this experimental arrangement excludes other effects known to cause photocurrents. ${ }^{24}$ The source of infrared radiation is a $Q$-switched $\mathrm{CO}_{2}$ laser with operating wavelengths $\lambda$ $=9.2-10.8 \mu \mathrm{m}$ (corresponding photon energies $\hbar \omega$
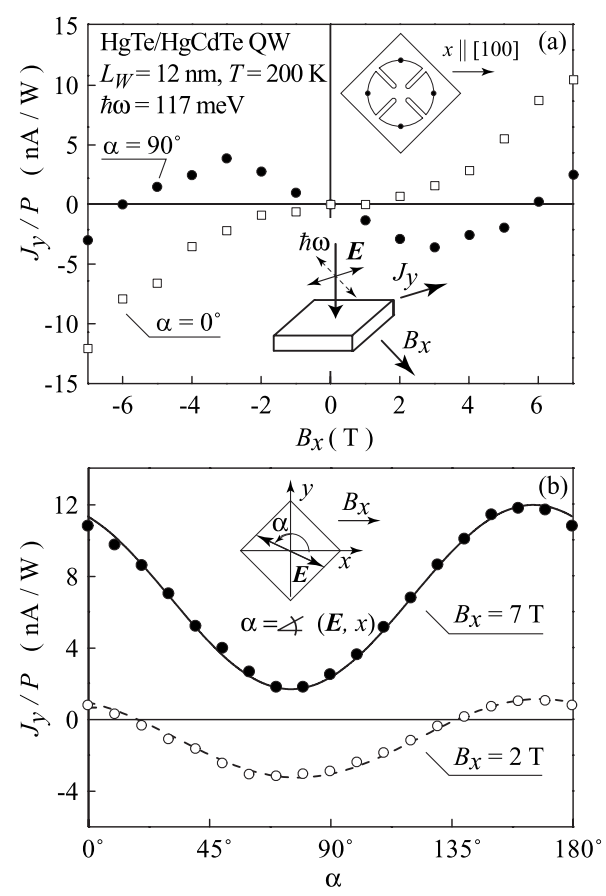

FIG. 1. Magnetic-field and polarization dependences of the photocurrent measured in a QW structure with the well width $L_{W}=12 \mathrm{~nm}$ at temperature $200 \mathrm{~K}$. Data are presented for normally incident mid-infrared radiation with $\hbar \omega=117 \mathrm{meV}$ $(\lambda=10.57 \mu \mathrm{m})$ and $P \approx 0.3 \mathrm{~kW}$. The magnetic field $\boldsymbol{B}$ is applied parallel to the $x$ axis and the photocurrent is measured in the direction $y$ normal to the vector $\boldsymbol{B}$. (a) Magnetic-field dependence for two states of polarization with the azimuth angle $\alpha$ equal to $0^{\circ}$ and $90^{\circ}$. (b) The dependence of the photocurrent on $\alpha$ measured for two magnetic-field strengths. The data on polarization dependence are fitted after Eqs. (1) and (6). The insets show the experimental geometry and the orientation of the light electric field $\boldsymbol{E}$ and the magnetic field $\boldsymbol{B}$ with respect to the sample orientation.

$=135-115 \mathrm{meV})$. In the investigated narrow gap QWs the radiation of these photon energies may induce interband optical transitions or transitions between size-quantized subbands. While the direct optical transitions dominate in the radiation absorption, the less intensive free-carrier absorption (Drude-type) may contribute substantially to the photocurrent generation. The radiation power $P$ was varied in the range from $10 \mathrm{~W}$ up to $1.2 \mathrm{~kW}$. For the measurements in the terahertz range we used molecular laser optically pumped by a TEA $\mathrm{CO}_{2}$ laser. ${ }^{31}$ With $\mathrm{NH}_{3}$ as active gas, 100 ns pulses of linearly polarized radiation with peak power $\sim 3 \mathrm{~kW}$ are obtained at wavelengths $\lambda=90,148$, and $280 \mu \mathrm{m}$ (corresponding photon energies $\hbar \omega$ are $13.7,8.4$, and $4.4 \mathrm{meV}$ ). We also used a $\mathrm{CH}_{3} \mathrm{~F}$ as active gas to obtain radiation with $\lambda$ $=496 \mu \mathrm{m}(\hbar \omega=2.5 \mathrm{meV})$. The photon energies in the terahertz range are smaller than the band gap as well as the size-quantized subband separation and at moderate temperatures terahertz radiation induces only free-carrier absorption in the lowest conduction subband. At low temperatures this radiation may also cause direct transitions due to ionization of impurities and transitions between spin-split subbands due to Zeeman or Rashba effects.

In our experiments we used the linearly polarized radiation. In order to vary the angle $\alpha$ between the light polariza- 
tion plane and the magnetic field, the plane of polarization of the radiation incident on the sample was rotated. Hereafter the angle $\alpha=0^{\circ}$ is chosen in such a way that the incident light polarization is directed along the $x$ axis, see inset in Fig. 1(b). In the terahertz range we used $\lambda / 2$ plates, which enabled us to change the azimuth angle $\alpha$ from $0^{\circ}$ to $180^{\circ}$ covering all possible orientations of the electric-field vector in the QW plane. In the mid-infrared range we applied a Fresnel rhomb converting the linearly polarized laser radiation into the circularly polarized radiation and placed an additional double-Brewster-window polarizer behind the rhomb. Rotation of the polarizer enabled us to tune the azimuth angle $\alpha$.

\section{EXPERIMENTAL RESULTS}

First, we discuss the results obtained with the midinfrared radiation. Irradiating samples at normal incidence we observe, for the in-plane magnetic field $\boldsymbol{B} \| x$, a photocurrent signal in the $y$ direction. The width of the current pulses is about $300 \mathrm{~ns}$ which corresponds to the infrared laser pulse duration. The signal linearly depends on the radiation power up to $P \approx 1.2 \mathrm{~kW}$, the highest power used in our midinfrared experiments. In Fig. 1(a) the magnetic-field dependence of the photocurrent is plotted for $\mathrm{HgTe} / \mathrm{HgCdTe} \mathrm{QW}$ structure with the well width of $12 \mathrm{~nm}$. The data are obtained at $T=200 \mathrm{~K}$ for two polarization states of the radiation with the electric field $\boldsymbol{E}$ of the light wave aligned parallel and perpendicularly to the magnetic field. In the both cases the signal is an odd function of $\boldsymbol{B}$. Its strength and behavior upon variation in $B$ depends, however, on the orientation of the radiation electric-field vector. Figure 1(b) shows the dependence of the photocurrent $J_{y}$ on the orientation of polarization plane specified by the angle $\alpha$. The data can be well fitted by the equation

$$
J_{y}\left(\alpha, B_{x}\right)=J_{0}\left(B_{x}\right)+J_{1}\left(B_{x}\right) \cos 2 \alpha+J_{2}\left(B_{x}\right) \sin 2 \alpha .
$$

Below we demonstrate that exactly these dependences follow from the theory. The measurements in the two fixed polarization directions allow us to extract two individual contributions: $^{32}$ the polarization-independent background and the amplitude of one of the polarization-dependent contributions, namely,

$$
J_{0}=\frac{J_{y}\left(0^{\circ}\right)+J_{y}\left(90^{\circ}\right)}{2}, \quad J_{1}=\frac{J_{y}\left(0^{\circ}\right)-J_{y}\left(90^{\circ}\right)}{2} .
$$

Figure 2 shows magnetic-field dependence of $J_{0}$ and $J_{1}$ for samples with the well widths of 8 and $22 \mathrm{~nm}$ at $T=200 \mathrm{~K}$. The signal behavior is different for these structures. We have found that, for the QW with $L_{W}=8 \mathrm{~nm}$, the photocurrent depends linearly on the magnetic field. On the other hand, in the QW with $L_{W}=22 \mathrm{~nm}$ the photocurrent can be described by a superposition of linear-in- $B$ and cubic-in- $B$ terms:

$$
J_{y}(B)=a B+b B^{3} .
$$

Figure 2 shows that the $B^{3}$ term is more pronounced in the polarization-independent photocurrent $J_{0}$. We focus below particularly on this photocurrent because our measurements
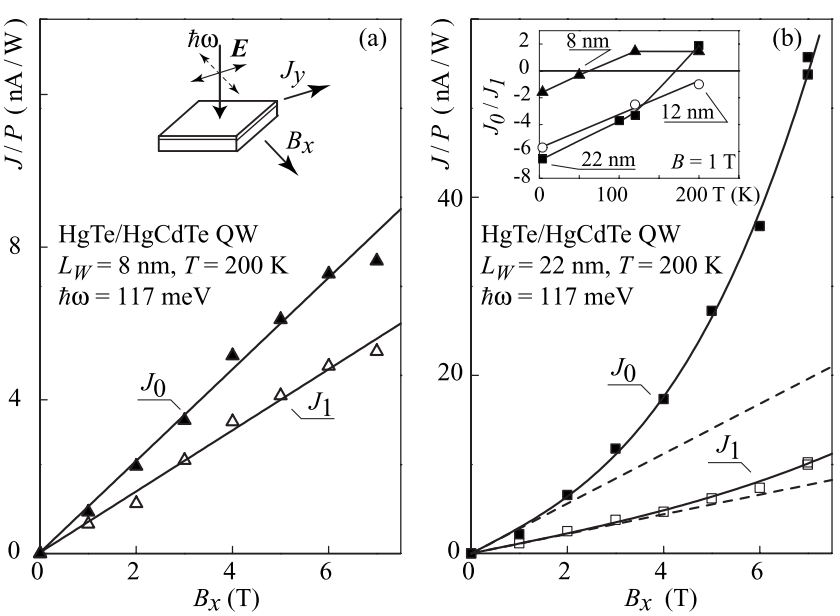

FIG. 2. Magnetic-field dependence of (a) the polarizationindependent photocurrent $J_{0}$ and (b) the polarization-dependent photocurrent $J_{1}$ obtained for the QW structures with $L_{W}=8$ and 22 $\mathrm{nm}$ at temperature $200 \mathrm{~K}$. Data are given for normally incident radiation of $P \approx 0.3 \mathrm{~kW}$ and the photon energy $\hbar \omega=117 \mathrm{meV}$. The data are fitted after Eqs. (3) and (6). For the QW structure with $L_{W}=8 \mathrm{~nm}$ the fitting is limited by linear terms. Dashed lines on the right panel demonstrate the linear contribution only. Insets show the experimental geometry and the temperature dependence of the ratio of polarization-independent and dependent photocurrents for QWs with $L_{W}=8,12$, and $22 \mathrm{~nm}$ at $B=1 \mathrm{~T}$.

reveal that this contribution dominates the photocurrent in the almost whole temperature range even at low magnetic fields, where the total photocurrent is mostly linear in $B$. While the linear dependence of the photocurrent on magnetic field is previously reported for various structures the observation of the cubic in magnetic-field photocurrent is unexpected and has not been detected so far. We emphasize that the last term in Eq. (3) corresponding to $J_{0}$ is strong and overcomes the linear-in- $B$ contribution at the magnetic field about $6 \mathrm{~T}$.

Similar behavior was observed in the structure with $L_{W}$ $=12 \mathrm{~nm}$. Moreover, in this sample the coefficients $a$ and $b$ for polarization-independent photocurrent $J_{0}$ have opposite signs resulting in a sign inversion observed for $B$ at about 4 T [see Fig. 3(a)]. In the structure with $L_{W}=5 \mathrm{~nm}$ the signals were too small to conclude definitely on the magnetic-field dependence (but it is measurable at the excitation with $\mathrm{THz}$ radiation). The decrease in temperature drastically affects the experimental data. At intermediate temperature of $120 \mathrm{~K}$ we have observed that the linear-in- $B$ contribution in QW with $L_{W}=22 \mathrm{~nm}$ changes its sign [see Fig. 3(b)]. Now, the sample with $L_{W}=22 \mathrm{~nm}$ also shows the sign inversion of the photocurrent $J_{0}$ with rising $B$, in the first sample with $L_{W}=8 \mathrm{~nm}$ the data are still well described by the linear-in- $B$ dependence. Further reduction in temperature to the liquid helium temperature results in the sign inversion of the linear-in- $B$ current in sample with $L_{W}=8 \mathrm{~nm}$ but also yields to the cubic-in- $B$ component [see Fig. 3(c)]. Now, the magneticfield dependence of the photocurrent in all samples is described by the linear- and cubic-in- $B$ terms with prefactors of opposite signs. The total current tends to the sign inversion, however, at substantially larger magnetic fields $B$. 

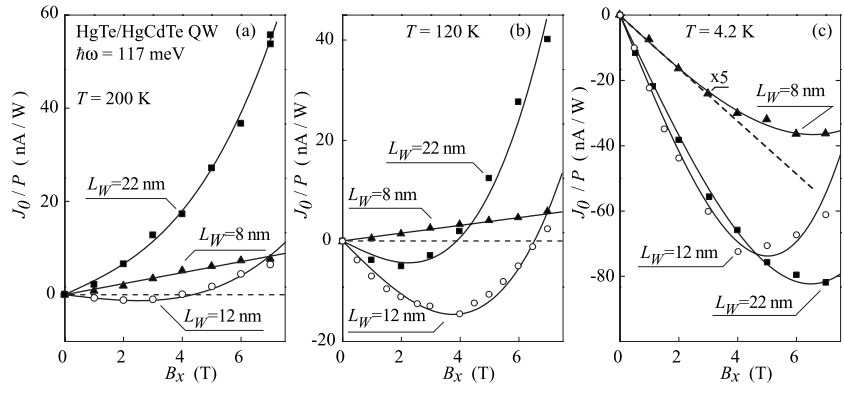

FIG. 3. Magnetic-field dependences of the polarizationindependent photocurrent $J_{0}$ obtained for QW structures at different temperatures. Data are given for normally incident radiation of $P$ $\approx 0.3 \mathrm{~kW}$ and the photon energy $\hbar \omega=117 \mathrm{meV}$. The photocurrent is measured in the direction perpendicular to $\boldsymbol{B}$ in QWs of three different widths. The data are fitted according to Eqs. (3) and (6). The dashed line in the panel (c) is plotted according to the linear law.

Now we turn to the experiments with terahertz radiation. We observed magnetic-field-induced photocurrent in all structures including sample with $L_{W}=5 \mathrm{~nm}$ and at all wavelengths used. Like in the mid-infrared range the signal depends on the radiation polarization (see Fig. 4) and is well described by Eq. (1). Figure 5(a) shows the magnetic-field dependence of the polarization-independent contribution to the photocurrent $J_{0}$ obtained in the wide $\mathrm{QW}$ with $L_{W}$ $=22 \mathrm{~nm}$ in response to the radiation of the photon energy $\hbar \omega=4.4 \mathrm{meV}(\lambda=280 \mu \mathrm{m})$. Figure 5(a) demonstrates that also in the terahertz range the photocurrent in the QW with $L_{W}=22 \mathrm{~nm}$ is well described by Eq. (3) with significant contribution of the cubic-in- $B$ term at high magnetic field. At low temperature we also detected a peak in the magneticfield dependence (a dip for absolute value of the signal). The peak has minimum at $B \approx 4 \mathrm{~T}$ and a half-width of about 0.75 T. Applying radiation of $496 \mu \mathrm{m}$ wavelength we obtained that the magnetic-field position of the peak linearly scales with the photon energy [Fig. 5(b)]. At shorter wavelength, e.g., with the photon energy $\hbar \omega=8.4 \mathrm{meV}(\lambda=148 \mu \mathrm{m})$, no peak has been detected at $B \leq 7 \mathrm{~T}$. Similar behavior is also detected in the polarization-dependent contribution $J_{1}$; how-

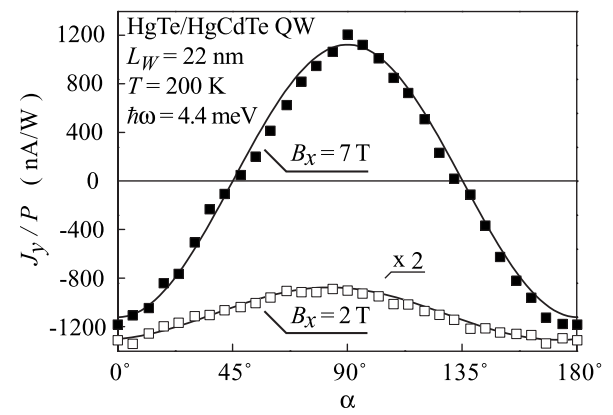

FIG. 4. Polarization dependence of the photocurrent $J_{y}$ excited by terahertz radiation in the QW structure with $L_{W}=22 \mathrm{~nm}$. The dependence is obtained at $T=200 \mathrm{~K}$, photon energy $\hbar \omega$ $=4.4 \mathrm{meV}(\lambda=280 \mu \mathrm{m})$, radiation power $P \approx 50 \mathrm{~W}$, and for two magnetic-field strengths. The full lines are the fits after Eqs. (1) and (6).

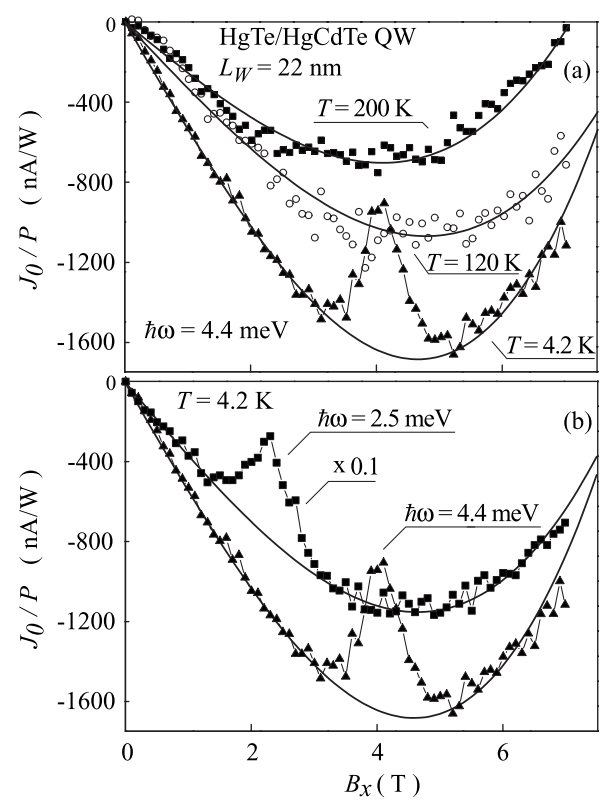

FIG. 5. Magnetic-field dependence of the polarizationindependent photocurrent $J_{0}$ excited by terahertz radiation in the QW structure with $L_{W}=22 \mathrm{~nm}$. (a) The photocurrent is measured in the direction perpendicular to $\boldsymbol{B}$ in response to the radiation of $\lambda$ $=280 \mu \mathrm{m}$ of $P \approx 50 \mathrm{~W}$ measured at three temperatures. (b) The photocurrent is measured at liquid helium temperature in response to the radiation of two photon energies. The lines are plotted according to Eqs. (3) and (6).

ever, the peak in this contribution is much less pronounced. Figure 6 demonstrates that linear-in- $B$ as well as cubic-in- $B$ current contributions $J_{0}$ and $J_{1}$ drastically increase with increasing of the wavelength. We see that at the longest wave-

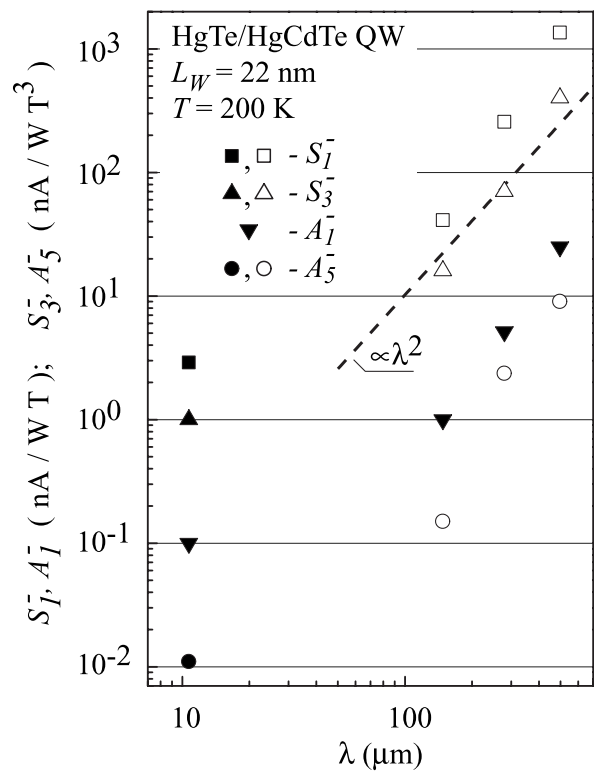

FIG. 6. Wavelength dependences of the absolute values of coefficients $S_{1}^{-}, S_{3}^{-}, A_{1}^{-}$, and $A_{5}^{-}$[see Eq. (6)] obtained for the QW structure with $L_{W}=22 \mathrm{~nm}$ at $T=200 \mathrm{~K}$. Full symbols correspond to negative values of the coefficients. The dashed line is plotted according to the wavelength square law. 


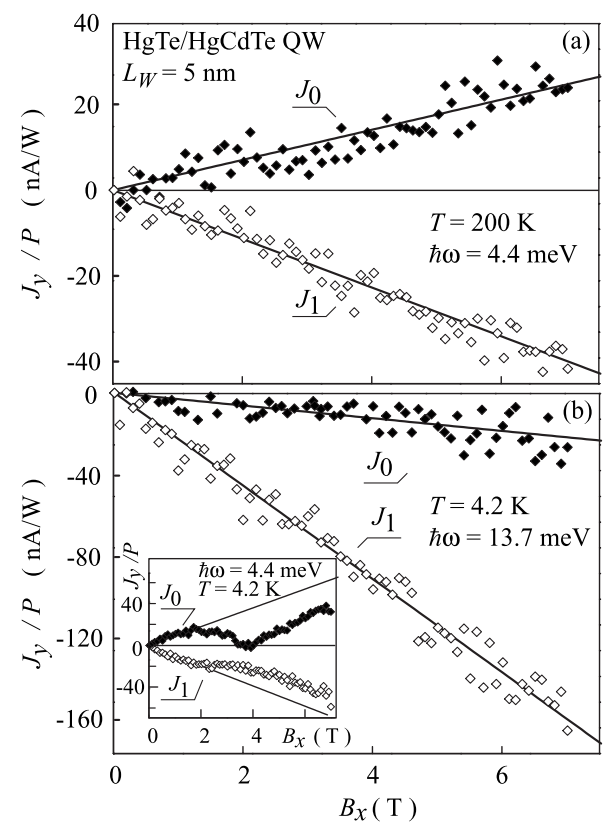

FIG. 7. Magnetic-field dependence of the photocurrent excited by terahertz radiation in the QW structure with $L_{W}=5 \mathrm{~nm}$. (a) The polarization-independent and polarization-dependent contributions to the photocurrent measured at $T=200 \mathrm{~K}$ in the direction perpendicular to $\boldsymbol{B}$ in response to the radiation with the photon energy $\hbar \omega=4.4 \mathrm{meV}(\lambda=280 \mu \mathrm{m})$ and $P \approx 3 \mathrm{~kW}$. (b) Photocurrent measured at liquid helium temperature in response to the radiation with the photon energy $\hbar \omega=13.7 \mathrm{meV}(\lambda=90 \mu \mathrm{m})$. The full lines are plotted according to Eqs. (3) and (6) with coefficients $b$ and $A_{1,5}^{-}$ equal to zero. The inset shows the data obtained at liquid helium temperature in response to the radiation with $\hbar \omega=4.4 \mathrm{meV}$.

length used $(\lambda=496 \mu \mathrm{m})$ all current contributions are more than two orders of magnitude larger than that detected in the mid-infrared range. We note that some contributions invert the sign with wavelength increasing.

In contrast to the wide QWs, in the narrowest QW sample $\left(L_{W}=5 \mathrm{~nm}\right.$ ) we observe that the photocurrent depends only linearly on the magnetic field $B$. This is demonstrated in Fig. 7(a) for both, polarization-independent and polarizationdependent, photocurrents obtained for $T=200 \mathrm{~K}$ and excitation with the photon energy $\hbar \omega=4.4 \mathrm{meV}$. The linear behavior of the photocurrent is observed even at low temperatures down to $4.2 \mathrm{~K}$ applying radiation of $\lambda=90 \mu \mathrm{m} \quad(\hbar \omega$ $=13.7 \mathrm{meV}$ ) wavelength [Fig. 7(b)]. For a longer wavelength this behavior is masked by the wide peak presented in the magnetic-field dependence of the photocurrent [see inset in Fig. 7(b)]. At the photon energy $\hbar \omega=4.4 \mathrm{meV}$ the peak position is close to that observed in the QW with $L_{W}$ $=22 \mathrm{~nm}$, but it is much wider and is characterized by a halfwidth of at least $3 \mathrm{~T}$. Like in the wide QWs, at higher photon energies no peak has been seen for $B \leq 7 \mathrm{~T}$ allowing one to analyze the magnetic-field dependence unaffected by the peak.

\section{PHENOMENOLOGY}

In order to describe the observed magnetic-field and polarization dependences, we first derive here phenomenologi- cal equations for the photocurrents in two-dimensional HgTe-based structures. Holding the linear and cubic in the magnetic-field strength $B$ terms, MPGE for unpolarized or linearly polarized radiation at normal incidence is given by

$$
\begin{aligned}
j_{\alpha}= & \sum_{\beta \gamma \delta} \phi_{\alpha \beta \gamma \delta} B_{\beta} \frac{e_{\gamma} e_{\delta}^{*}+e_{\delta} e_{\gamma}^{*}}{2} I \\
& +\sum_{\beta \mu \nu \gamma \delta} \Xi_{\alpha \beta \mu \nu \gamma \delta} B_{\beta} B_{\mu} B_{\nu} \frac{e_{\gamma} e_{\delta}^{*}+e_{\delta} e_{\gamma}^{*}}{2} I .
\end{aligned}
$$

Here $\phi$ and $\Xi$ are a fourth- and a sixth-rank pseudotensor, respectively, being symmetric in the last two indices, $e_{\gamma}$ are components of the unit vector of light polarization, and $I$ is the light intensity. We note that while in the theoretical consideration the current density $\boldsymbol{j}$ is used, in the experiments the electric current $\boldsymbol{J}$ is measured which is proportional to the current density $\boldsymbol{j}$.

We consider (001)-oriented HgTe-based QWs. Depending on the equivalence or nonequivalence of the QW interfaces their symmetry may belong to one of the point groups $D_{2 d}$ or $C_{2 v}$, respectively. The present experiments have been carried out on the asymmetric structures of $C_{2 v}$ symmetry and, therefore, here we will focus on these QWs only. For the $C_{2 v}$ point group it is convenient to write the components of the magnetophotocurrent in the coordinate system with $x^{\prime}\left\|[1 \overline{1} 0], y^{\prime}\right\|[110]$, and $z \|[001]$ being the growth direction. The advantage of this system is that the in-plane axes $x^{\prime}$ and $y^{\prime}$ lie in the crystallographic planes (110) and (1히) which are the mirror reflection planes containing the twofold axis $C_{2} \| z$.

In QWs of $C_{2 v}$ symmetry class the tensors $\phi$ and $\Xi$ have, respectively, six and 12 linearly independent components and in the system $x^{\prime}, y^{\prime}$, and $z$ for normal incidence of the linearly polarized or unpolarized light and the in-plane magnetic field Eq. (4) is reduced to

$$
\begin{aligned}
j_{x^{\prime}}= & I\left[S_{1} B_{y^{\prime}}+S_{2} B_{y^{\prime}}\left(\left|e_{x^{\prime}}\right|^{2}-\left|e_{y^{\prime}}\right|^{2}\right)+S_{3} B_{x^{\prime}}\left(e_{x^{\prime}} e_{y^{\prime}}^{*}+e_{y^{\prime}} e_{x^{\prime}}^{*}\right)\right] \\
& +I B_{y^{\prime}}\left[A_{1} B^{2}+A_{2}\left(B_{x^{\prime}}^{2}-B_{y^{\prime}}^{2}\right)\right] \\
& +I B_{y^{\prime}}\left[A_{3} B^{2}+A_{4}\left(B_{x^{\prime}}^{2}-B_{y^{\prime}}^{2}\right)\right]\left(\left|e_{x^{\prime}}\right|^{2}-\left|e_{y^{\prime}}\right|^{2}\right) \\
& +I B_{x^{\prime}}\left[A_{5} B^{2}+A_{6}\left(B_{x^{\prime}}^{2}-B_{y^{\prime}}^{2}\right)\right]\left(e_{x^{\prime}} e_{y^{\prime}}^{*}+e_{y^{\prime}} e_{x^{\prime}}^{*}\right), \\
j_{y^{\prime}}= & I\left[S_{1}^{\prime} B_{x^{\prime}}+S_{2}^{\prime} B_{x^{\prime}}\left(\left|e_{x^{\prime}}\right|^{2}-\left|e_{y^{\prime}}\right|^{2}\right)+S_{3}^{\prime} B_{y^{\prime}}\left(e_{x^{\prime}} e_{y^{\prime}}^{*}+e_{y^{\prime}} e_{x^{\prime}}^{*}\right)\right] \\
& +I B_{x^{\prime}}\left[A_{1}^{\prime} B^{2}+A_{2}^{\prime}\left(B_{x^{\prime}}^{2}-B_{y^{\prime}}^{2}\right)\right] \\
& +I B_{x^{\prime}}\left[A_{3}^{\prime} B^{2}+A_{4}^{\prime}\left(B_{x^{\prime}}^{2}-B_{y^{\prime}}^{2}\right)\right]\left(\left|e_{x^{\prime}}\right|^{2}-\left|e_{y^{\prime}}\right|^{2}\right) \\
& +I B_{y^{\prime}}\left[A_{5}^{\prime} B^{2}+A_{6}^{\prime}\left(B_{x^{\prime}}^{2}-B_{y^{\prime}}^{2}\right)\right]\left(e_{x^{\prime}} e_{y^{\prime}}^{*}+e_{y^{\prime}} e_{x^{\prime}}^{*}\right) .
\end{aligned}
$$

Here $S_{i}$ and $A_{j}$ are linearly independent components of the tensors $\phi$ and $\Xi$, respectively. The polarization dependence of the photocurrent is determined by the factors $\left(\left|e_{x^{\prime}}\right|^{2}\right.$ $\left.-\left|e_{y^{\prime}}\right|^{2}\right)$ and $\left(e_{x^{\prime}} e_{y^{\prime}}^{*}+e_{y^{\prime}} e_{x^{\prime}}^{*}\right)$. 
In our experiments the magnetic field was oriented along the cubic axis $\boldsymbol{B} \| x$ and the current $J_{y}$ was measured perpendicularly to $\boldsymbol{B}$. For this experimental geometry Eq. (5) reduces to

$$
\begin{aligned}
j_{y}= & I B_{x}\left(-S_{1}^{-}+S_{2}^{-} \sin 2 \alpha-S_{3}^{-} \cos 2 \alpha\right) \\
& +I B_{x}^{3}\left(-A_{1}^{-}+A_{3}^{-} \sin 2 \alpha-A_{5}^{-} \cos 2 \alpha\right),
\end{aligned}
$$

where $S_{l}^{-}=\left(S_{l}-S_{l}^{\prime}\right) / 2, A_{l}^{-}=\left(A_{l}-A_{l}^{\prime}\right) / 2$, and $\alpha$ is an angle between the linear polarization direction and the axis $x \|[100]$; see inset of Fig. 1(b). Thus, for the polarization-independent and polarization-dependent contributions to the photocurrent measured in the experiment we have $J_{0} \propto-\left(B_{x} S_{1}^{-}+B_{x}^{3} A_{1}^{-}\right), J_{1}$ $\propto-\left(B_{x} S_{3}^{-}+B_{x}^{3} A_{5}^{-}\right)$, and $J_{2} \propto\left(B_{x} S_{2}^{-}+B_{x}^{3} A_{3}^{-}\right)$.

Equation (6) describes well the macroscopic features of the photocurrent. In accordance with the experimental data it contains both linear- and cubic-in- $B$ contributions and fully describes the observed polarization dependence [see Figs. 1(b) and 4]. Figure 2 shows that in the field $B \leq 1 \mathrm{~T}$ the linear part is the dominant one and it reveals both polarization-independent $J_{0}$ and polarization-dependent $J_{1}$ parts. According to Eq. (6) they are given by the coefficients $S_{1}^{-}$and $S_{3}^{-}$, respectively. The temperature dependence of the ratio $J_{0} / J_{1}=S_{1}^{-} / S_{3}^{-}$is presented in the inset of Fig. 2(b) and shows that polarization-independent contribution dominates the total photocurrent over almost the entire temperature range. In the narrowest QW with $L_{W}=5 \mathrm{~nm}$ and in QW with $L_{W}=8 \mathrm{~nm}$ at high temperature the linear-in- $B$ behavior remains up to the highest magnetic fields applied. In other samples, by contrast, for $B>1$ T the cubic-in- $B$ contribution is clearly detected and even dominates the photocurrent.

\section{BAND STRUCTURE AND OPTICAL TRANSITIONS}

Now we calculate the band structure of our samples and indicate optical transitions responsible for radiation absorption and the MPGE current generation. HgTe as a bulk material is a zero-gap semimetal, whereas a narrow energy gap opens up in a quantum well. Depending on the actual well width and temperature, the band structure is either normal or inverted. In the latter case, the ordering of the subbands in the QW is reversed compared to common semiconductors.

In Fig. 8 the calculated band structure of $8 \mathrm{~nm} \mathrm{QW}$ is shown together with possible direct optical transitions corresponding to the photon energy $\hbar \omega=117 \mathrm{meV}$ used in the experiment with mid-infrared radiation. The band structure of (001)-grown $\mathrm{HgTe} / \mathrm{Hg}_{0.3} \mathrm{Cd}_{0.7} \mathrm{Te} \mathrm{QW}$ was calculated using the eight-band $\boldsymbol{k} \cdot \boldsymbol{p}$ model in envelope function approximation. ${ }^{33}$ This QW is a type-III heterostructure (see insets of Fig. 8) that causes mixing of the electron states and strong coupling between the conduction and valence bands. In order to take into account the coupling and the resulting nonparabolicity of the bands the Kane model with the usual eight-band basis set $\left\{\left|u_{n}^{0}\right\rangle\right\}=\left|\Gamma_{6}, \pm 1 / 2\right\rangle,\left|\Gamma_{8}, \pm 1 / 2\right\rangle$, $\left|\Gamma_{8}, \pm 3 / 2\right\rangle,\left|\Gamma_{7}, \pm 1 / 2\right\rangle$ was used. Assuming the basis functions $u_{n}^{0}$ to be the same throughout the heterostructure and using the correct operator ordering in the effective-mass Hamiltonian for the eight-component envelope function vector in accordance with the envelope function theory ${ }^{34}$ the
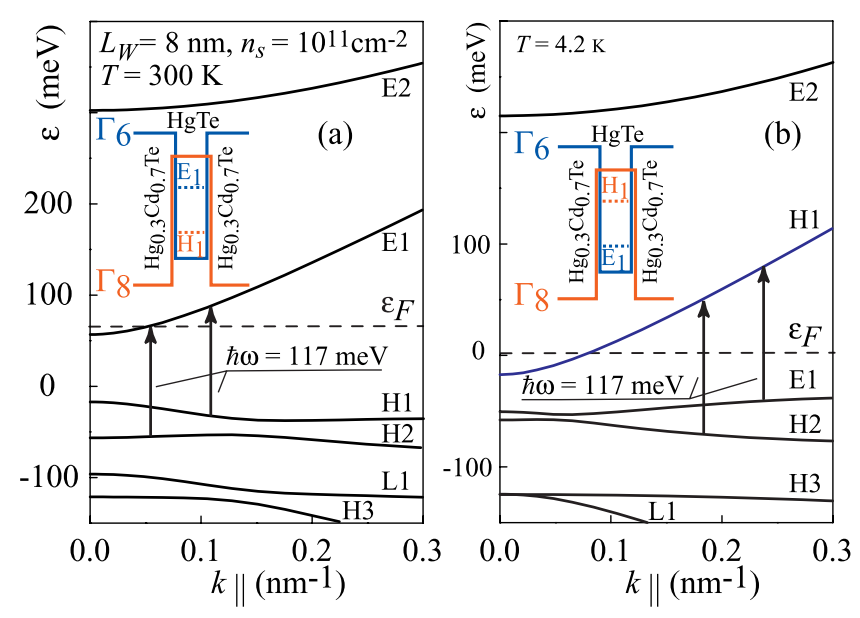

FIG. 8. (Color) Calculated band structure for $8 \mathrm{~nm} \mathrm{QW} \mathrm{at} \mathrm{(a)}$ $300 \mathrm{~K}$ and (b) $4.2 \mathrm{~K}$. Arrows show optical transitions induced by mid-infrared radiation used in the experiments $(\hbar \omega=117 \mathrm{meV})$. Insets sketch the band profile of $\mathrm{HgTe}$-based QWs with noninverted (left panel) and inverted (right panel) band structure.

boundary conditions at material interfaces are automatically satisfied. The total eight-band Hamiltonian $H$ of the QW system is given by $H_{0}+H_{1}+H_{2}+H^{\mathrm{BP}}+V(z)$, where $H_{0}$ is the diagonal contribution including the band-edge potentials for the chosen basis set $\left\{\left|u_{n}^{0}\right\rangle\right\}, H_{1}$ and $H_{2}$ describe the direct coupling between the bands of this basis set and their coupling via remote bands in the second-order perturbation theory, respectively, $H^{\mathrm{BP}}$ is the Bir-Pikus Hamiltonian describing the effects of strain in the structure, and $V(z)$ is the self-consistently calculated Hartree potential. The electron wave function is expanded in terms of the $\Gamma$-point Blochfunction basis,

$$
\Psi(\boldsymbol{r})=\sum_{n} F_{n}(\boldsymbol{r})\left|u_{n}^{0}\right\rangle,
$$

where $F_{n}(\boldsymbol{r})=\exp \left[i\left(k_{x} x+k_{y} y\right)\right] f_{n}(z)$ are the envelope functions, $k_{x}$ and $k_{y}$ are the wave vector components in the plane of the QW, and $n$ is the index enumerating eight states $\Gamma_{6}, \Gamma_{8}$, and $\Gamma_{7}$. Then the Schrödinger equation for $\Psi$ is transformed to the following system of coupled differential equations for the envelope functions and the energy levels near $k=0$ :

$$
\begin{gathered}
\sum_{n^{\prime}} H_{n n^{\prime}} f_{n^{\prime}}(z)=E f_{n}(z), \\
H_{n n^{\prime}}=E_{n^{\prime}}(z) \delta_{n n^{\prime}}+\sum_{\alpha} P_{n n^{\prime}}^{\alpha} k_{\alpha}+\sum_{\alpha, \beta} k_{\alpha} D_{n n^{\prime}}^{\alpha \beta} k_{\beta}+H_{n n^{\prime}}^{\mathrm{BP}} \\
+V(z) \delta_{n n^{\prime}} .
\end{gathered}
$$

Here, the summation is performed over the index $n^{\prime}$ of the chosen basis set and $\alpha, \beta=x, y, z ; k_{z}=-i \partial / \partial z, E_{n^{\prime}}(z)$ are the respective band-edge potentials, the momentum matrix elements $P_{n n^{\prime}}^{\alpha}$ describe the coupling between the $n$ and $n^{\prime}$ Bloch states exactly, and the $D_{n n^{\prime}}^{\alpha \beta}$ elements take into account the band coupling via remote bands. Detailed description of the model and explicit form of the Hamiltonian as well as the 
band-structure parameters employed in the calculations are given in Ref. 33.

The system of eight coupled differential equations (8) of the second order for the envelope function components was transformed then into a matrix eigenvalue problem by means of the expansion of the envelope function components in terms of the complete basis set which results in the required convergence for type-III heterostructures. The subbands in Fig. 8 are labeled as heavy-hole- $(H i)$, electron- $(E i)$, and light-hole-like $(\mathrm{Li})$ in accordance with the properties of the corresponding wave functions at $k_{\|}=0$; see Ref. 35 .

We emphasize that HgTe-based QW may have a normal or inverted band structure depending on its width and the temperature. ${ }^{21}$ For example, for $T=4.2 \mathrm{~K}$ and QW width $L_{W} \lesssim 6 \mathrm{~nm}$ electronlike (holelike) subbands form the conduction (valence) band. Figure 8 shows that while at $4.2 \mathrm{~K}$ heterostructures with $8 \mathrm{~nm}$ QWs are characterized by the inverted band structure, at room temperature it has a normal band structure. At $T=4.2 \mathrm{~K}$ for $L_{W} \gtrsim 6 \mathrm{~nm} H 1$ subband lies above the $E 1$ subband and becomes the lowest conduction subband [see Fig. 8(b)]. With temperature increasing the critical width is shifted to larger values and for $T=300 \mathrm{~K}$ and $L_{W}=8 \mathrm{~nm}$ the QW has a normal sequence of the subbands. Calculations of the band structure for QWs with $L_{W}$ $=12$ and $22 \mathrm{~nm}$ demonstrate that they have an inverted band structure for all temperatures used in our experiments, whereas QW of $5 \mathrm{~nm}$ width has a noninverted band structure in the whole temperature range. The analysis of the band structure of investigated samples reveals that the nonlinear behavior of the MPGE is detected only in samples having inverted band structure.

Our calculations show that mid-infrared radiation with the photon energy on the order of $100 \mathrm{meV}$ used in experiments causes in all our samples direct interband optical transitions (see Fig. 8). The photon energies of applied terahertz radiation $(\hbar \omega=3-14 \mathrm{meV})$ are much smaller than the energy gap and intersubband separation, therefore this radiation causes only indirect (Drude-type) optical transitions. At low temperatures with $k_{\mathrm{B}} T<\hbar \omega$ terahertz radiation may also cause ionization of impurities, intraimpurity transitions, or direct transitions between the Zeeman spin-split subbands. These mechanisms may have a resonancelike behavior and be responsible for peaks observed in the magnetic-field dependences of the photocurrent at liquid helium temperature. A comparatively large width of these peaks covering several tesla indicates that they are most probably due to impurityrelated mechanisms. The magnetic field shifts the band edge as well as the impurity level and tunes the binding energy to the photon energy making the direct optical excitation possible. The mechanism of this additional channel of the radiation absorption and the resulting MPGE are out of scope of this paper.

\section{MICROSCOPIC MODELS AND DISCUSSION}

The most surprising result obtained in the experiment is that in samples with $L_{W}=12 \mathrm{~nm}$ and $L_{W}=22 \mathrm{~nm}$, as well as in the sample with $L_{W}=8 \mathrm{~nm}$ at low temperature, the cubic-in- $B$ contribution to $J$ is strong and may overcome the
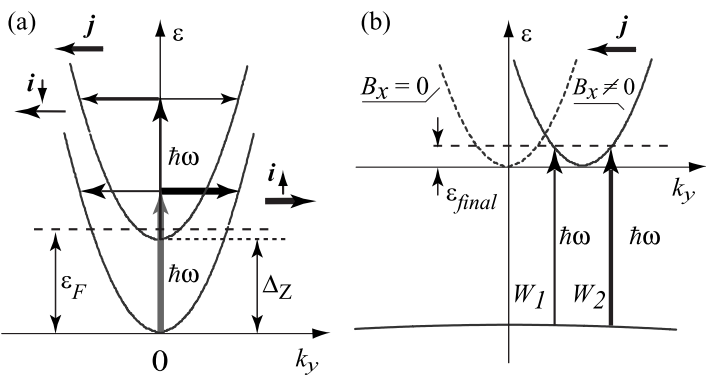

FIG. 9. Microscopic models of MPGE (a) due to imbalance of the spin photocurrents in the in-plane magnetic field and (b) due to the diamagnetic mechanism at direct intersubband transitions.

linear-in- $B$ contribution at the magnetic field of $4-5 \mathrm{~T}$. Therefore, we focus below on possible microscopic mechanisms of such a nonlinear behavior which is observed in QWs with the inverted band structures only. Since to the best of our knowledge the band structure of $\mathrm{HgTe} / \mathrm{HgCdTe} \mathrm{QWs}$ in in-plane magnetic fields is not available, we perform here only a qualitative microscopic analysis of the effect.

First we discuss the terahertz spectral range where radiation absorption is dominated by Drude-type processes. In this case, the photocurrent is mainly caused by asymmetry of the electron scattering by phonons and static defects in the magnetic field. ${ }^{30}$ Such a magnetic-field-induced scattering asymmetry can be of both spin-dependent and diamagnetic (spinindependent) origins. The spin-dependent mechanism of MPGE comes from the imbalance of the spin photocurrents in the in-plane magnetic field. ${ }^{24}$ Microscopically, it is based on spin-dependent scattering which accompanies the freecarrier absorption. Figure 9(a) sketches the indirect optical transitions within two spin subbands. Vertical arrows indicate optical transitions from the initial state $k_{y}=0$ while the horizontal arrows describe a scattering event to a final state with either positive or negative electron wave vector $k_{y}^{\prime}$. Due to the spin dependence of scattering the transitions to states with positive and negative $k_{y}^{\prime}$ occur with unequal probabilities. This is indicated by horizontal arrows of different thicknesses. By that the free-carrier absorption leads to a pure spin current where particles with opposite spin orientations flow in opposite directions. Similarly to the excitation mechanism, energy relaxation of electron gas heated by Drude absorption, also involving electron scattering, is asymmetric and yields spin separation as well. By application of an external magnetic field which polarizes free carriers, the spin photocurrent is converted into an electric current proportional to the Zeeman splitting for small fields. We note that the mechanism based on asymmetry of the photoexcitation yields polarization-dependent photocurrent while that related to asymmetry of energy relaxation results in polarization-independent signal. ${ }^{24}$ In QWs with inverted band structure the ground conduction subband, which is populated in equilibrium, is formed from the $\Gamma_{8}$-band states [see Fig. 8(b)]. The Zeeman splitting $\Delta_{Z}$ of heavy-hole states in the in-plane magnetic field depends strongly nonlinear on $B$ (Ref. 36). Since for this mechanism $j(\boldsymbol{B}) \propto \Delta_{\mathrm{Z}}(\boldsymbol{B})$, the photocurrent exhibits a nonlinear behavior in the magnetic field. In the narrow $\mathrm{QW}$ with $L_{W}=5 \mathrm{~nm}$ the ground conduction 
subband is formed from the $\Gamma_{6}$-band states. Here the Zeeman splitting is linear in $B$ and a noticeable cubic-in- $B$ contribution to the photocurrent is absent as observed in our experiments.

The diamagnetic mechanism of the MPGE under freecarrier absorption is also related to asymmetry of electron scattering in the magnetic field. ${ }^{26,28}$ However the asymmetry stems from the magnetic-field-induced mixture of states from different quantum subbands, which is not related to the Zeeman splitting. This mixture is more efficient for subbands formed from the same Bloch states (i.e., between $E 1$ and $E 2$ or $H 1$ and $H 2)$ and determined by the ratio of $\hbar e B /\left(m^{*} c\right)$ to the intersubband energy separation $\Delta \varepsilon$, where $m^{*}$ is the inplane effective mass. In QWs with inverted band structure the ground $H 1$ subband is close to the valence subbands of hole type; see Fig. 8(b). Therefore in moderate magnetic fields of several tesla, the ratio becomes not small, which leads to nonlinear dependence of the photocurrent on magnetic field. By contrast, in the structure with normal band arrangement, Fig. 8(a), the energy separation between the ground subbands $E 1$ and $E 2$ exceeds $200 \mathrm{meV}$ and the MPGE current linearly depends on $B$.

The mechanisms described above may also be responsible for the photocurrent caused by mid-infrared radiation. Although the contribution from the Drude processes to the total absorption does not seem to be dominant in the spectral range where interband transitions are possible, it may nevertheless determine the photocurrent. This scenario is supported by the drastic spectral dependence of the photocurrent demonstrated in Fig. 6. Indeed, the photocurrent strength increases by more than an order of magnitude with increasing wavelength, the dependence usually detected for Drude-type absorption. Another contribution may come from the direct intersubband transitions caused by mid-infrared radiation. Such a mechanism of MPGE is proposed in Ref. 25 and is based on magnetic-field-induced shift of quantum subbands in $\boldsymbol{k}$ space which is described by the linear-in- $\boldsymbol{B}$ contribution to the electron energy given by $\delta E_{\boldsymbol{k}}^{(\nu)}=\left(e \hbar \bar{z}_{\nu} / \mathrm{cm}^{*}\right)[\boldsymbol{B} \times \boldsymbol{k}]_{z}$, where $\bar{z}_{\nu}$ is the mean coordinate along the growth direction and $\nu$ is the QW subband index. The energy spectrum of the QW, including the diamagnetic shift, is sketched in Fig. 9(b). In conventional QW structures with parabolic dispersion of the valence and conduction subbands, the relative subband shift leads to a photocurrent at direct optical transitions. ${ }^{25,27}$ Indeed, in such systems due to the energy and momentum conservation the points of optical transitions are shifted in the $\boldsymbol{k}$ space resulting in asymmetric distribution of photoexcited carriers with respect to the subbands minima, i.e., to a magnetic-field-induced photocurrent. However, this straightforward mechanism of MPGE gets ineffective in HgTe-based structures under study where the valence subbands are flat (see Fig. 8). In this particular case, the points of optical transitions remain symmetric with respect to the conduction subband minimum. Therefore, the diamagnetic shift of the conduction subband does lead to a photocurrent only if the probability of optical transitions $W$ depends on the wave vector. Such a dependence may come from mixture of the states at finite in-plane wave vector resulting in $W=w_{0}+w_{2} k^{2}$. This term together with the diamagnetic shift of the conduction subband gives rise to the photocurrent $j(B) \propto w_{2} B$; see Fig. 9(b). Moreover, in QWs with inverted band structure and closely spaced valence subbands, like in the case of wide HgTe QWs, one can expect that the parameter $w_{2}$ can be large enough and the magnetic field has a remarkable effect on $w_{2}$. This leads to a nonlinear dependence of the photocurrent on magnetic field.

\section{SUMMARY}

In conclusion, we have studied magnetogyrotropic photogalvanic effect in $n$-doped (001)-grown $\mathrm{HgTe} / \mathrm{HgCdTe} \mathrm{QWs}$. The intrasubband (Drude-type) and interband absorption of infrared/terahertz radiation in the heterostructures is shown to cause a dc electric current in the presence of an in-plane magnetic field. The photocurrent dependences on the radiation polarization state, magnetic-field strength, temperature, and QW widths are studied. The macroscopic features of the photocurrent are in good agreement with the phenomenological description of MPGE based on the symmetry arguments. As an important result, we have observed that, in quantum wells with the inverted band structure, the MPGE photocurrent becomes strongly nonlinear with the rising magnetic field. This is in contrast to all previous studies on MPGE carried out on III-V-based heterostructures, as well as to our results obtained on $\mathrm{HgTe} / \mathrm{HgCdTe} \mathrm{QWs}$ with normal band ordering, in which the MPGE current is shown to be a linear function of the magnetic field $B$. This observation allows one to conclude on the normal or inverted band ordering at any temperature and in an easy way, by mere measuring of the magnetic-field dependence of the MPGE. The experimental results are analyzed in terms of the microscopic models of magnetogyrotropic photogalvanic effect based on asymmetry of optical transitions or carrier relaxation in the $\boldsymbol{k}$ space. This effect is related to the gyrotropic properties of the structures. It is shown that both intrasubband and interband absorption of radiation may lead to spin-related as well as spinindependent photocurrents if an external magnetic field is applied in the plane of the quantum well. One of the most probable scenarios of the observed nonlinearity is based on the cubic-in- $B$ Zeeman splitting of the lowest conduction subband. To prove this statement additional experiments, e.g., electron spin resonance investigations, are needed. Further access to the origin of the photocurrent and various mechanisms contributing in its formation might be provided by investigation of the photocurrent temperature and spectral behavior and, in particular, of the current sign inversions.

\section{ACKNOWLEDGMENTS}

We thank M. M. Voronov for helpful discussions. The financial support of the DFG via programs GA 501/6-3 and AS327/2-1 as well as support of RFBR is gratefully acknowledged. E.L.I. thanks the Merkator Program of the DFG for support and L.E.G. acknowledges the "Dynasty" Foundation-ICFPM and President grant for young scientists. 
${ }^{1}$ H.-A. Engel, E. I. Rashba, and B. I. Halperin, Handbook of Magnetism and Advanced Magnetic Materials (John Wiley \& Sons, Chichester, UK, 2007).

${ }^{2}$ Y. A. Bychkov and E. I. Rashba, Pis'ma Zh. Eksp. Teor. Fiz. 39, 66 (1984) [JETP Lett. 39, 78 (1984)].

${ }^{3}$ S. D. Ganichev and W. Prettl, J. Phys.: Condens. Matter 15, R935 (2003) and references cited therein.

${ }^{4}$ W. Zawadzki and P. Pfeffer, Semicond. Sci. Technol. 19, R1 (2004).

${ }^{5}$ R. Winkler, Handbook of Magnetism and Advanced Magnetic Materials (John Wiley \& Sons, New York, 2007).

${ }^{6}$ J. Fabian, A. Matos-Abiague, C. Ertler, P. Stano, and I. Zutic, Acta Phys. Slov. 57, 565 (2007).

${ }^{7}$ Spin Physics in Semiconductors, edited by M. I. Dyakonov (Springer, Berlin, 2008).

${ }^{8}$ J. Hinz, H. Buhmann, M. Schäfer, V. Hock, C. R. Becker, and L. W. Molenkamp, Semicond. Sci. Technol. 21, 501 (2006).

${ }^{9}$ Y. S. Gui, C. R. Becker, N. Dai, J. Liu, Z. J. Qiu, E. G. Novik, M. Schäfer, X. Z. Shu, J. H. Chu, H. Buhmann, and L. W. Molenkamp, Phys. Rev. B 70, 115328 (2004).

${ }^{10}$ A. Pfeuffer-Jeschke, F. Goschenhofer, S. J. Cheng, V. Latussek, J. Gerschütz, C. R. Becker, R. R. Gerhardts, and G. Landwehr, Physica B 256-258, 486 (1998).

${ }^{11}$ Z. D. Kvon, S. N. Danilov, N. N. Mikhailov, S. A. Dvoretsky, and S. D. Ganichev, Physica E 40, 1885 (2008).

${ }^{12}$ M. von Truchsess, V. Latussek, C. R. Becker, and E. Batke, J. Cryst. Growth 159, 1104 (1996).

${ }^{13}$ C. R. Becker, C. Brüne, M. Schäfer, A. Roth, H. Buhmann, and L. W. Molenkamp, Phys. Status Solidi C 4, 3382 (2007).

${ }^{14}$ V. Daumer, I. Golombek, M. Gbordzoe, E. G. Novik, V. Hock, C. R. Becker, H. Buhmann, and L. W. Molenkamp, Appl. Phys. Lett. 83, 1376 (2003).

${ }^{15}$ X. C. Zhang, K. Ortner, A. Pfeuffer-Jeschke, C. R. Becker, and G. Landwehr, Phys. Rev. B 69, 115340 (2004).

${ }^{16}$ S. N. Danilov, B. Wittmann, P. Olbrich, W. Eder, W. Prettl, L. E. Golub, E. V. Beregulin, Z. D. Kvon, N. N. Mikhailov, S. A. Dvoretsky, V. A. Shalygin, N. Q. Vinh, A. F. G. van der Meer, B. Murdin, and S. D. Ganichev, J. Appl. Phys. 105, 013106 (2009).

${ }^{17}$ B. Wittmann, S. N. Danilov, Z. D. Kvon, N. N. Mikhailov, S. A. Dvoretsky, R. Ravash, W. Prettl, and S. D. Ganichev, in Narrow Gap Semiconductors 2007, Proceedings of the 13th International Conference, Springer Proceedings in Physics Vol. 119, edited by B. N. Murdin and S. K. Clowes (Springer, Dordrecht, 2008), p. 7.

${ }^{18}$ J. Liu, V. Daumer, Y. S. Gui, V. Hock, C. R. Becker, H. Buhmann, and L. W. Molenkamp, J. Supercond. 16, 365 (2003).
${ }^{19}$ Y. S. Gui, C. R. Becker, J. Liu, V. Daumer, V. Hock, H. Buhmann, and L. W. Molenkamp, Europhys. Lett. 65, 393 (2004).

${ }^{20}$ M. König, S. Wiedmann, C. Brüne, A. Roth, H. Buhmann, L. W. Molenkamp, X.-L. Qi, and S.-C. Zhang, Science 318, 766 (2007).

${ }^{21}$ M. König, H. Buhmann, L. W. Molenkamp, T. Hughes, C.-X. Liu, X.-L. Qi, and S.-C. Zhang, J. Phys. Soc. Jpn. 77, 031007 (2008).

${ }^{22}$ V. Lechner, L. E. Golub, P. Olbrich, S. Stachel, D. Schuh, W. Wegscheider, V. V. Bel'kov, and S. D. Ganichev, Appl. Phys. Lett. 94, 242109 (2009).

${ }^{23}$ V. V. Bel'kov, S. D. Ganichev, E. L. Ivchenko, S. A. Tarasenko, W. Weber, S. Giglberger, M. Olteanu, H.-P. Tranitz, S. N. Danilov, Petra Schneider, W. Wegscheider, D. Weiss, and W. Prettl, J. Phys.: Condens. Matter 17, 3405 (2005).

${ }^{24}$ S. D. Ganichev, V. V. Bel'kov, S. A. Tarasenko, S. N. Danilov, S. Giglberger, Ch. Hoffmann, E. L. Ivchenko, D. Weiss, W. Wegscheider, Ch. Gerl, D. Schuh, J. Stahl, J. De Boeck, G. Borghs, and W. Prettl, Nat. Phys. 2, 609 (2006).

${ }^{25}$ A. A. Gorbatsevich, V. V. Kapaev, and Yu. V. Kopaev, Pis'ma Zh. Eksp. Teor. Fiz. 57, 565 (1993) [JETP Lett. 57, 580 (1993)].

${ }^{26}$ O. V. Kibis, Zh. Eksp. Teor. Fiz. 115, 959 (1999) [JETP 88, 527 (1999)].

${ }^{27}$ H. Diehl, V. A. Shalygin, S. N. Danilov, S. A. Tarasenko, V. V. Bel'kov, D. Schuh, W. Wegscheider, W. Prettl, and S. D. Ganichev, J. Phys.: Condens. Matter 19, 436232 (2007).

${ }^{28}$ S. A. Tarasenko, Phys. Rev. B 77, 085328 (2008).

${ }^{29}$ V. V. Bel'kov, P. Olbrich, S. A. Tarasenko, D. Schuh, W. Wegscheider, T. Korn, C. Schüller, D. Weiss, W. Prettl, and S. D. Ganichev, Phys. Rev. Lett. 100, 176806 (2008).

${ }^{30}$ V. V. Bel'kov and S. D. Ganichev, Semicond. Sci. Technol. 23, 114003 (2008)

${ }^{31}$ S. D. Ganichev and W. Prettl, Intense Terahertz Excitation of Semiconductors (Oxford University Press, New York, 2006).

${ }^{32}$ We note that both polarization-dependent components of the total photocurrent in Eq. (1) stem from the same microscopic mechanism; see Ref. 23.

${ }^{33}$ E. G. Novik, A. Pfeuffer-Jeschke, T. Jungwirth, V. Latussek, C. R. Becker, G. Landwehr, H. Buhmann, and L. W. Molenkamp, Phys. Rev. B 72, 035321 (2005).

${ }^{34}$ M. G. Burt, J. Phys.: Condens. Matter 4, 6651 (1992); 11, R53 (1999); B. A. Foreman, Phys. Rev. B 48, 4964 (1993).

${ }^{35}$ M. von Truchsess, A. Pfeuffer-Jeschke, V. Latussek, C. R. Becker, and E. Batke, J. Cryst. Growth 184/185, 1190 (1998).

${ }^{36} \mathrm{R}$. Winkler, Spin-Orbit Coupling Effects in Two-Dimensional Electron and Hole Systems, Springer Tracts in Modern Physics Vol. 191 (Springer, Berlin, 2003). 